\title{
Rule-Based Activity Recognition in Ambient Intelligence
}

\author{
Grigoris Antoniou \\ Institute of Computer Science, FORTH-ICS, and \\ Department of Computer Science, University of Crete, Crete, Greece \\ antonioulics.forth.gr
}

\begin{abstract}
Activity recognition is an important, multi-faceted problem with a broad application scope. In this talk we will present a rule-based activity recognition system with the option of using confidence values in our rules and facts. Each activity definition rule has some primary and some optional events and the absence of any of the optional events, only decreases the confidence value of the recognized complex event. We recognize all possible complex events (activities) based on predefined rules, which express temporal and spatial combinations of atomic and complex events. Then we detect all conflicted events (recognized events that overlap but use common resources). The optimal solution is found with an optimization function that takes into account complex event's confidence, temporal duration and number of used atomic events. Adjusting this function, results in higher or lower abstraction levels in our results (more generic events with bigger duration / more specific events with lower duration).

As application domain we use ambient assisted living (e.g. for elderly persons). The approach has been implemented, and tested in a real ambient intelligence environment hosted by the FORTH Institute of Computer Science.
\end{abstract}

